\title{
Non-linear description of massive neutrinos in the framework of large-scale structure formation
}

\author{
Hélène Dupuy ${ }^{1,2}$ \\ ${ }^{1}$ Institut de Physique Théorique, CEA, IPhT, URA 2306 du CNRS, F-91191 Gif-sur-Yvette, \\ France \\ ${ }^{2}$ Institut d'Astrophysique de Paris, UMR 7095 du CNRS, Université Pierre et Marie Curie, 98 \\ bis bd Arago, 75014 Paris, France \\ email: helene.dupuy@cea.fr ; dupuy@iap.fr
}

\begin{abstract}
There is now no doubt that neutrinos are massive particles fully involved in the nonlinear growth of the large-scale structure of the universe. A problem is that they are particularly difficult to include in cosmological models because the equations describing their behavior in the non-linear regime are cumbersome and difficult to handle. In this manuscript I present a new method allowing to deal with massive neutrinos in a very simple way, based on basic conservation laws. This method is still valid in the non-linear regime. The key idea is to describe neutrinos as a collection of single-flow fluids instead of seeing them as a single hot multi-flow fluid. In this framework, the time evolution of neutrinos is encoded in fluid equations describing macroscopic fields, just as what is done for cold dark matter. Although valid up to shell-crossing only, this approach is a further step towards a fully non-linear treatment of the dynamical evolution of neutrinos in the framework of large-scale structure growth.
\end{abstract}

Keywords. cosmology: theory and large-scale structure of universe, neutrinos

\section{Introduction}

Observational cosmology has been particularly fruitful recently, as evidenced by the great success of the Planck mission (Planck Collaboration 2013). From the theoretical point of view, such an achievement naturally opens perspectives. Any component of the universe involved in large-scale structure formation has to be examined minutely. In particular, the discovery of the massiveness of neutrinos has triggered a considerable effort in theoretical and numerical cosmology to infer the impact of this mass on the evolution of cosmological perturbations.

The first study in which massive neutrinos are properly treated in the linear theory of gravitational perturbations dates back from 1994 (Ma and Bertschinger 1994). Then the connection between neutrino masses and cosmology has been investigated in full detail (Lesgourgues and Pastor 2006). To a large extent, current and future cosmology projects aim at exploiting this connection to put constraints on neutrino masses. Such surveys are sensitive to the non-linear growth of structure, whence the importance of studying the non-linear regime in cosmological perturbation theory. In this manuscript, I summarize the results of a study in which the authors design analytical tools to explore the impact of massive neutrinos on large-scale structure growth within the non-linear regime (Dupuy and Bernardeau 2014). 


\section{The standard linear description of neutrinos}

The strategy usually adopted to describe neutrinos, massive or not, is calqued on that used to describe the radiation fluid: neutrinos are considered as a single hot multistream fluid whose evolution is dictated by the behavior of its distribution function in phase-space $f$. The key equation is the Boltzmann equation. For neutrinos, contrary to radiation, it is taken in the collisionless limit since neutrinos do not interact with ordinary matter (neither at the time of recombination nor after). It leads to the Vlasov equation, $\frac{\mathrm{d} f}{\mathrm{~d} \eta}=0$, where $\eta$ is a time coordinate.

The standard linear description of neutrinos is based i) on a decomposition of the phase-space distribution function into a homogeneous part and a first-order inhomogeneous contribution in the Vlasov equation and ii) on a decomposition of the latter into harmonic functions. It leads to the standard Boltzmann hierarchy. Finally, relevant physical quantities can be built out of the coefficients of the harmonic decomposition (see e.g. Ma and Bertschinger 1994). This approach is powerful and largely used in the litterature. But, unfortunately, it is valid in the linear regime only.

To get a non-linear description, which is essential to do precision cosmology, one can e.g compute the fully non-linear moments of the Vlasov equation (Van de Rijt 2012). Although interesting because fully non-linear, the resulting hierarchy is unfortunately very difficult to handle. As a matter of fact, the non-linear equations one can find in the litterature to describe neutrinos are generally not exploitable because of their complexity. An analytical description of neutrinos in the non-linear regime is therefore still missing.

\section{The non-linear description of cold dark matter, a model to follow}

Cold dark matter is described as a collection of identical point particles, non-relativistic and sensitive to gravitational interaction only (for notations and more details, see Bernardeau 2013). Using the Newtonian approximation, its time evolution is encoded in the Vlasov-Poisson system, which leads to the continuity and Euler equations

$$
\begin{gathered}
\frac{\partial \delta(\mathbf{x}, t)}{\partial t}+\frac{1}{a}\left[(1+\delta(\mathbf{x}, t)) u_{i}(\mathbf{x}, t)\right]_{, i}=0 \\
\frac{\partial u_{i}(\mathbf{x}, t)}{\partial t}+\frac{\dot{a}}{a} u_{i}(\mathbf{x}, t)+\frac{1}{a} u_{j}(\mathbf{x}, t) u_{i}(\mathbf{x}, t)_{, j}=-\frac{1}{a} \Phi(\mathbf{x}, t)_{, i}-\frac{\left(\rho(\mathbf{x}, t) \sigma_{i j}(\mathbf{x}, t)\right)_{, j}}{a \rho(\mathbf{x}, t)} .
\end{gathered}
$$

A major simplification comes from the single-flow approximation, which consists in neglecting the velocity dispersion term $\sigma_{i j}$ because, in a cold fluid, it is extremely small compared to the velocity gradients induced by density fluctuations. This assumption breaks down as soon as shell-crossing starts. Very little analytical results are known in presence of shell-crossing so description is then purely numerical.

Besides, the Euler equation shows that, in the single-flow approximation, the velocity field is a gradient. Equations can thus be much simplified when introducing the velocity divergence $\theta$. Those two specificities of cold dark matter allow to write the motion equations on a compact form (see Bernardeau 2013 for notations),

$$
\frac{\partial \Psi_{a}(\mathbf{k}, \eta)}{\partial \eta}+\Omega_{a}^{b}(\eta) \Psi_{b}(\mathbf{k}, \eta)=\gamma_{a}^{b c}\left(\mathbf{k}_{1}, \mathbf{k}_{2}\right) \Psi_{b}\left(\mathbf{k}_{1}, \eta\right) \Psi_{c}\left(\mathbf{k}_{2}, \eta\right)
$$

where $\Psi_{a}(\mathbf{k}, \eta) \equiv(\delta(\mathbf{k}, \eta),-\theta(\mathbf{k}, \eta))$. It makes the writing of a formal solution possible

$$
\Psi_{a}(\mathbf{k}, \eta)=g_{a}^{b}(\eta) \Psi_{b}\left(\mathbf{k}, \eta_{0}\right)+\int_{\eta_{0}}^{\eta} \mathrm{d} \eta^{\prime} g_{a}^{b}\left(\eta, \eta^{\prime}\right) \gamma_{b}^{c d}\left(\mathbf{k}_{1}, \mathbf{k}_{2}\right) \Psi_{c}\left(\mathbf{k}_{1}, \eta^{\prime}\right) \Psi_{d}\left(\mathbf{k}_{2}, \eta^{\prime}\right)
$$


This is a very good starting point for the development of theories allowing to establish a connection with some interesting observables. The formalism that has to be developed to do this is explained in detail in Bernardeau 2013. It is now well-established and it can be considered as a model to follow when it comes to study the impact of neutrinos on large-scale structure formation in the non-linear regime.

\section{A non-linear alternative to the standard description of neutrinos}

The leading idea of Dupuy and Bernardeau 2014 is to describe neutrinos as a collection of single-flow fluids instead of considering them as a single multi-flow fluid in order to take advantage of the single-flow approximation. This approximation can not be applied directly to the overall neutrino fluid because, unlike cold dark matter, there is no reason why velocity dispersion should be small for neutrinos. To circumvent the problem, the overall neutrino fluid is splitted into several flows in this study. Each flow, or each neutrino fluid, is defined as the collection of all the particles that have a given initial velocity. Such fluids are thus actually single-flow fluids and remain so until shell-crossing starts. In practice, it starts when neutrino velocities become low enough for gravity to make neutrinos turn around. So, at that time, neutrinos are not relativistic anymore and behave as cold dark matter. Describing this phenomenon is beyond the scope of this study. So far, it is also beyond the scope of the study of cold dark matter.

The first equation of motion is simply the conservation of the number of particles in each neutrino fluid, which gives in a perturbed Friedmann-Lemaitre metric (written in the conformal Newtonian gauge)

$$
\partial_{\eta} n-(1+2 \phi+2 \psi) \partial_{i}\left(\frac{P_{i}}{P_{0}} n\right)=3 n\left(\partial_{\eta} \phi-\mathcal{H}\right)+n\left(2 \partial_{i} \psi-\partial_{i} \phi\right) \frac{P_{i}}{P_{0}},
$$

where $n$ is the proper number density and $P_{i}$ is the comoving momentum field. To obtain this, only linear terms in the metric perturbations $\psi$ and $\phi$ have been taken into account. Indeed, observations demonstrate that what matters in the non-linear regime is not metric-metric coupling but the non-linear growth of the fields. Moreover, for a single-flow fluid, the conservation of the energy-momentum tensor combined with the conservation of the number of particles gives an equation for $P_{i}$ only,

$$
\partial_{\eta} P_{i}-(1+2 \phi+2 \psi) \frac{P_{j}}{P_{0}} \partial_{j} P_{i}=P_{0} \partial_{i} \psi+\frac{P_{j} P_{j}}{P_{0}} \partial_{i} \phi
$$

This equation shows in particular that the linear part of $P_{i}$ is a gradient. This property is interesting since the fact that the velocity field is a gradient is one of the key ingredients of the cold dark matter description. The set of equations (4.1)-(4.2) is a relativistic generalization of the motion equations of cold dark matter. It is fully equivalent to the non-linear moments of the Boltzmann equation (Van de Rijt 2012). The advantage is that it is much more manageable in the non-linear regime than an infinite non-linear hierarchy. Some numerical tests have been realized in order to compare this approach to the standard one. The goal was to compute the overall (i.e. integrated over all the neutrino fluids) multipole energy distribution and to check that it gives the same values as integration of the linear Boltzmann hierarchy. Calculations are thus perfomed in the linear regime only. As illustrated on Fig.1, the agreement between both approaches is extremely good. By its specificity, the multi-fluid approach allows also to show the convergence of the number density contrast and of the velocity divergence of each flow to the ones of the cold dark matter component. As an illustration of this phenomenon, the time evolution of the velocity divergences of several neutrino fluids is presented on Fig. 2. 

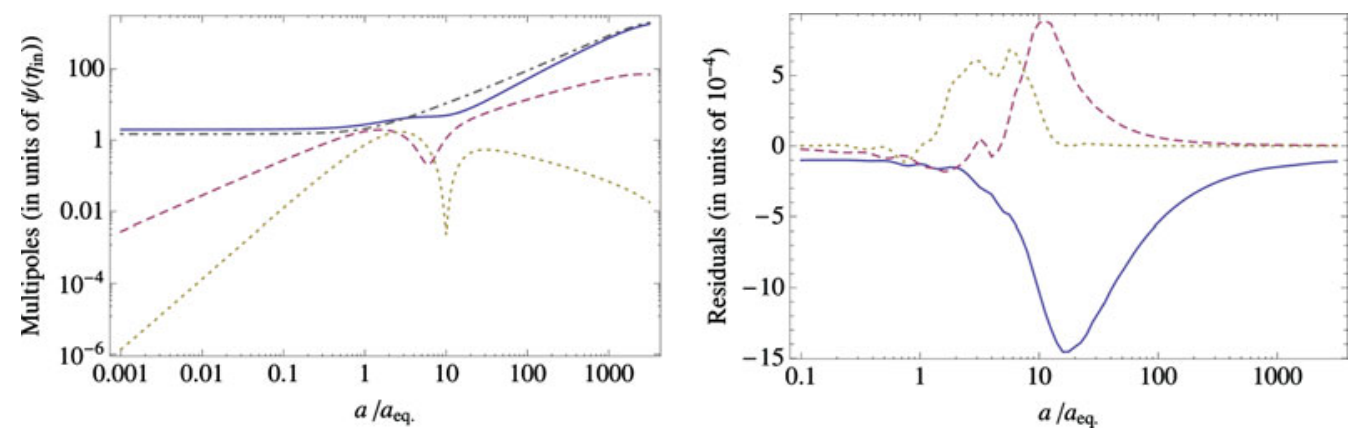

Figure 1. Time evolution of the energy density contrast (solid line), velocity divergence (dashed line) and shear stress (dotted line) of the neutrinos. The dot-dashed line is presented for comparison and corresponds to the density contrast of the dark matter component. Left panel: the quantities are computed with the multi-fluid approach. Right panel: residuals (defined as the relative differences) when the two methods are compared. Numerical integration has been done with 40 neutrino fluids, $k$ is set to $k_{\text {eq }} \approx 0.01 h / \mathrm{Mpc}$ and the neutrino mass $m$ is set to $0.3 \mathrm{eV}$.
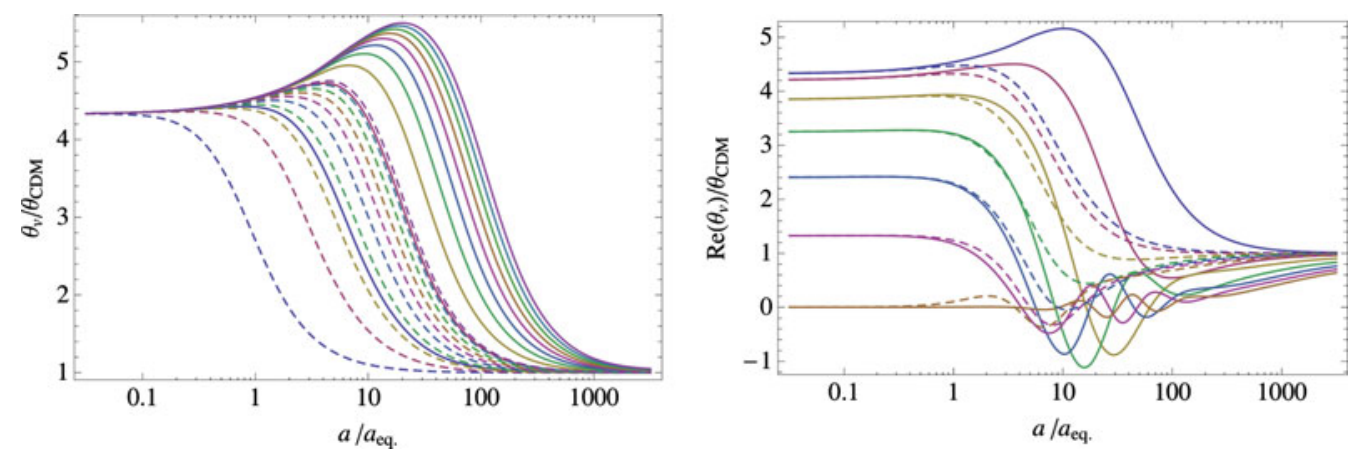

Figure 2. Time evolution of the velocity divergence. Left panel: values of the initial velocity moduli, denoted $\tau$, range from $0.45 k_{B} T_{0}$ (bottom lines) to $9 k_{B} T_{0}$ (top lines) with $\mu=0$ ( $\mu$ being the cosine of the angle between the initial velocity vector and the wave vector). Right panel is for $\tau=3.6 k_{B} T_{0}$ and $\mu$ ranging from $\mu=0$ (top lines) to $\mu=1$ (bottom lines). The time evolution of the velocity divergence of each flow is plotted in units of the dark matter velocity divergence. The wave number is set to $k_{\text {eq }}$, the solid lines correspond to a $0.05 \mathrm{eV}$ neutrino mass and the dashed lines to a $0.3 \mathrm{eV}$ neutrino mass.

\section{References}

Bernardeau, F. 2013, ArXiv e-prints, 1311.2724

Dupuy, H. \& Bernardeau, F. 2014, JCAP, 1:30

Lesgourgues, J. \& Pastor, S. 2006, Phys. Rept., 429:307-379

Ma, C.-P. \& Bertschinger, E. 1994, ApJ, 429:22-28

Planck Collaboration 2013, ArXiv e-prints, 1303.5062

Planck Collaboration 2013, ArXiv e-prints, 1303.5076

Van de Rijt, N. 2012, PhD thesis "Signatures of the primordial universe in large-scale structure surveys", Ecole Polytechnique \& Institut de Physique Théorique, CEA Saclay 